\title{
RADIORÉSISTANCE EN RELATION AVEC L'ACTIVATION D'ONCOGÈNE
}

\author{
John B. LITTLE \\ Laboratory of Radiobiology \\ Harvard School of Public Health, \\ Boston, MA 02115, U.S.A.
}

Despite considerable research over the past three decades, the factors that control the response of human tumors to radiation remain poorly understood. Normal human cell strains studied in vitro show a rather narrow range in radiosensitivity (1), whereas human tumor cells vary considerably in their sensitivity to the cytotoxic effects of radiation $(2,3)$. Cells from some types of tumors such as soft tissue sarcomas are quite radiosensitive in vitro while a number of cell lines established from various human tumors including squamous cell carcinomas are unusually radioresistant, well beyond the range observed in normal cells (3). The reasons for this variation are not known.

Mutations in a number of different oncogenes and tumor suppressor genes have been associated with different types of human cancer. Two observations have suggested a possible role for such genes in the sensitivity of cells to killing by ionizing radiation. First, certain human diploid fibroblast cell strains transformed by the SV-40 virus were found to be less radiosensitive than their non-transformed counterparts. Second, evidence has been presented that transfection of rodent cells with an activated ras oncogene may render these cells more resistant to killing by radiation $(4,5)$.

We have studied these two phenomena systematically in normal human diploid fibroblasts, as well as in two human tumor cell lines. Three human diploid cell strains were transfected with an activated Ha-ras oncogene (EJ ras) or the SV-40 T-antigen (6). Multiple clones were isolated from each strain and examined for their sensitivity to $\mathrm{x}$-irradiation in vitro, as well as for morphological alterations, immortalization and tumorigenicity in nude mice. Cells transfected with the activated ras oncogene alone showed no morphological alterations nor significant changes in radiosensitivity. Clones expressing SV-40 T-antigen, on the other hand, were significantly radioresistant as compared with their parental cells or clones tranfected with the neo gene only. This radioresistant phenotype persisted in post-crisis, immortalized cell lines. These data suggest that expression of the SV-40 T-antigen but not activated Ha-ras plays a significant role in the radiosensitivity of human diploid cells. The radioresistant phenotype was not related to the enhanced level of genetic instability seen in pre-crisis and newly immortalized cells, nor to the process of immortalization itself. 
In order to examine the mechanism for this effect, experiments were designed to determine which of the various functional domains of the SV-40 T-antigen gene were involved in the radioresistance phenotype (7). A series of human diploid fibroblast cell clones were generated by DNA transfection with a group of SV-40 T-antigen mutants defective in these various functional domains. Cell clones transfected with mutants defective in nuclear localization or origin binding showed increased radioresistance similar to clones transfected with the wild-type gene. These clones also expressed morphological changes characteristic of SV-40 T-transfected cells. A mutant defective in its ability to bind the retinoblastoma protein showed moderately increased radioresistance. However, cell clones transfected with three different p53 bindingdefective mutant genes showed no change in radiosensitivity as compared with neo gene transfected control clones. Transfection with $T$-antigen mutants defective in either the retinoblastoma $(\mathrm{RB})$ or $\mathrm{p} 53$ binding domain yielded no morphological alterations characteristic of transformation. These data suggest that the $S V-40 \mathrm{~T} / \mathrm{p} 53$ complex may be of importance in the radioresistance phenotype.

In order to confirm this observation, cells were transfected with the E6 and E7 genes of the human papilloma virus (HPV18). The E6 gene controls p53 binding while the E7 gene controls binding of the retinoblastoma protein. Cells transfected with E6 and E7 or with E6 alone developed the radioresistant phenotype, consistent with the involvement of p53 in this process. Cells transfected with E7 alone became senescent, and clones could not be isolated for further study. We have therefore initiated experiments in which the intact retinoblastoma gene has been transfected into cell lines defective in RB expression. Preliminary results suggest that RB expression alone is not a major determinant of radiosensitivity in human cells.

The experiments described above suggest a role for the p53 gene product in the control of the radiosensitivity of normal human cells. An obvious question is whether this gene influences the sensitivity of human tumor cells or the response of human cancer to radiation therapy. Among a recently reported series of $\mathbf{3 0}$ tumor cell lines established from patients with squamous cell carcinoma of the head and neck, $16(53 \%)$ had mutations in p53 (8). Of these patients, 18 showed failure of local control of the tumor by radiation therapy. These radioresistant tumors were equally distributed among those showing normal and mutated p53. Furthermore, there is no clear correlation between p53 status and radiosensitivity of human tumor cell lines in vitro.

In order to further investigate these latter phenomena, two cell lines established from human squamous cell carcinomas were transfected with the SV-40 T-antigen. These tumor cell lines were chosen as one of them is quite radiosensitive, while the other is highly radioresistant. In neither case did transfection with SV-40 T-antigen significantly enhance the radioresistance of these cells as compared with neo-transfected control clones. Transfection with activated ras also had no significant effect on the radiosensitivity of the two human tumor cell lines.

Thus, while p53 expression appears to influence the radiosensitivity of normal cells, its effect on tumor cells is not clear. We hypothesize that tumor cells have undergone a number of mutagenic events in various oncogenes and tumor suppressor 
genes that have allowed them to escape normal growth controls and at the same time become refractory to the effects of p53 expression on radiosensitivity.

\section{References:}

1. Little, J.B. and J. Nove. Sensitivity of human diploid fibroblast cell strains from various genetic disorders to acute and protracted radiation exposure. Radiation Res. 123: 87-92, 1990.

2. Dahlberg, W.K., J.B. Little, J.A. Fletcher, H.D. Suit and P. Okunieff. Radiosensitivity in vitro of human soft tissue sarcoma cell lines and skin fibroblasts derived from the same patients. Int. J. Radiat. Biol. (in press, 1992).

3. Weichselbaum, R.R., W. Dahlberg and J.B. Little. Inherently radioresistant cells exist in some human tumors. Proc. Natl. Acad. Sci. (USA) 82: 4732-4735, 1985.

4. Fitzgerald, T.J., C. Daugherty, K. Kase, L. Rothstein, and J. Greenberg, Activated human $\mathrm{N}$-ras oncogene enhances $\mathrm{X}$-irradiation repair of mammalian cells in vitro less effectively at low dose rate. Amer. J. Clinical Oncology 8: 517-522, 1985.

5. Sklar, M.D. The ras oncogenes increase the intrinsic resistance of NIH3T3 cells to ionizing radiation. Science 239: 645-647, 1988.

6. Su, L.-N. and J.B. Little. Transformation and radiosensitivity of human diploid skin fibroblasts transfected with activated RAS oncogene and SV40 T-antigen. Int. J. Radiat. Biol. 62: 201-210, 1992.

7. Su, L.-N. and J.B. Little. Transformation and radiosensitivity of human diploid skin fibroblasts transfected with SV40 T-antigen mutants defective in RB and p53 binding domains. Int. J. Radiat. Biol. 62: 461-468, 1992.

8. Brachman, D.G., D. Graves, E. Vokes, M. Beckett, D. Haraf, A. Montag, E. Dunphy, R. Mick, D. Yandell and R.R. Weichselbaum. Occurrence of p53 gene deletions and human papilloma virus infection in human head and neck cancer. Cancer Res. 52: 4832-4836, 1992. 